\title{
Cell proliferation and invasion ability of human choriocarcinoma cells lessened due to inhibition of Sox2 expression by microRNA-145
}

\author{
FUHUI XU, HAIPENG WANG, XIA ZHANG, TE LIU and ZHIXUE LIU \\ School of Life Science and Technology, Tongji University, Shanghai 200092, P.R. China
}

Received June 26, 2012; Accepted October 10, 2012

DOI: $10.3892 / \mathrm{etm} .2012 .781$

\begin{abstract}
To date, the mechanism underlying the development of human choriocarcinomas has not been elucidated. It is hypothesized that the Sox 2 protein plays a pivotal role in the proliferation and invasion capacity of tumor cells. A microRNA (miR-145) was cloned and used to study the expression of Sox 2 and its regulatory effect on the proliferation and invasion capacity of the human choriocarcinoma cell line JAR. In the present study, Sox 2 mRNA and protein expression decreased in JAR and JEG-3 cells following transfection with the miR-145 expression virus. Cell proliferation assays indicated that miR-145 expression affected cell cycle regulation and suppressed the proliferation of choriocarcinoma cells in vitro. In addition, xenograft experiments confirmed the suppression of tumor growth in vivo due to cell cycle arrest. Therefore, endogenous mature miR-145 expression may have an important role in the pathogenesis of human choriocarcinomas via interference with the Sox 2 target gene by epigenetic modification. This information is of potential significance for the identification of therapeutic targets in human choriocarcinoma.
\end{abstract}

\section{Introduction}

Human choriocarcinomas, which are a rare form of cancer that develops in the uterus from tissue that would normally become the placenta, are a trophoblastic gestational disease. These carcinomas have been studied largely to investigate conditions associated with pregnancy, such as preeclampsia (1). However, the mechanism underlying choriocarcinoma development remains to be elucidated.

Correspondence to: Dr Zhixue Liu or Dr Te Liu, School of Life Science and Technology, Tongji University, 123 Siping Road, Shanghai 200092, P.R. China

E-mail: liuzhixue@163.com

E-mail: liute79@yahoo.com

Key words: choriocarcinoma, microRNA-145, Sox2, proliferation, invasion
microRNAs (miRNAs) are small RNA molecules (21-23 nt) that act as negative regulators of gene expression, either by blocking mRNA translation into protein or by RNA interference $(2,3)$. Previous studies have revealed that dysregulation of specific miRNAs is associated with certain types of cancer and are considered to act either as oncogenes or tumor suppressors, depending on the target gene (2,4-10). Examples include, miR-15a, which has been associated with chronic lymphocytic leukemia $(2,5,6)$, and also miR-21 and miR-17, which are upregulated, while miR-143 and miR-145 are downregulated in colorectal cancer $(11,12)$. The presence of the let-7 family of miRNAs is a prognostic factor in lung cancer $(5,6)$ and miR-182 inhibits the proliferation and invasion ability of the human lung adenocarcinoma cells via its effect on human cortical actin-associated protein (13). To date, investigation of the decreased expression of miR-199b in human choriocarcinoma by Chao et al indicated that epigenetic mechanisms play an important role in increasing the expression levels of protein phosphatase $2 \mathrm{~A}$ inhibitor and contribute to the pathogenesis of human choriocarcinoma (14). However, no studies have linked miR-145 expression with the proliferation and invasion capacity of human choriocarcinoma cells (2).

The transcription activator Sox 2 was originally studied in the context of sexual determination during the development of Drosophila embryos and thus, its name is an acronym for 'sex determination Y-box2' (15-17). Numerous studies have indicated a primary role for Sox 2 factor in the maintenance of embryonic stem cell pluripotency, and in later stages of development, in the repression of trophectoderm and epiblast genes. In addition, Sox 2 appears to have a significant role in the differentiation of the nervous system (16). Extensive studies have indicated that Oct 4 , Sox 2 and Nanog are required for self-renewal and pluripotency of embryonic stem cells $(17,18)$. Investigation of the expression and methylation profiles of Sox2 in placentas and gestational trophoblastic disease by Li et al indicated that epigenetic mechanisms play an important role in the transcriptional regulation of Sox 2 and contribute to the pathogenesis of gestational trophoblastic disease (19). By contrast, Xu et al reported that endogenous miR-145 represses the 3'-untranslated regions (3'-UTRs) of Oct4, Sox 2 and Klf4, and that increased miR-145 expression inhibits human embryonic stem cell self-renewal, represses expression of pluripotency genes and induces lineage-restricted differentia- 
tion (18). In addition, Sox 2 was closely associated with certain tumors, its inappropriate activation being involved in the development processes of human tumors, including the abnormal methylation modification of the promoter region of the Sox 2 gene. Nakatsugawa et al (20) analyzed the functions of Sox2 in cancer stem-like cells/cancer-initiating cells derived from human lung adenocarcinoma. Nakatsugawa et al revealed that the Sox 2 protein was detected in $>80 \%$ of cancer stem-like cells/cancer-initiating cells in primary lung carcinoma tissues. However, Sox 2 mRNA knockdown of the human lung cancer stem-like cells/cancer-initiating cells by gene-specific siRNA eliminated tumorigenicity in vitro and in vivo. These observations indicate that Sox 2 has a role in the maintenance of stemness and tumorigenicity of human lung carcinoma and is a potential target for treatment.

In view of this evidence, in the current study, the miR-145 expression vector was transfected into the human choriocarcinoma cell lines JAR and JEG-3 to determine its specific role in Sox 2 regulation and inhibition of cell proliferation, and invasion. These results are of potential importance for the identification of therapeutic targets in human choriocarcinoma.

\section{Materials and methods}

Cell lines and animals. The human choriocarcinoma cell lines JAR and JEG-3 were grown in DMEM (Hyclone, Logan, UT, USA) supplemented with $10 \%$ fetal bovine serum (PAA Laboratories Inc., Queensland, Australia), penicillin (100 U/ml), streptomycin $(100 \mathrm{U} / \mathrm{ml})$ and $2 \mathrm{mM}$ L-glutamine (all were purchased from Hyclone). The JAR cells were incubated at $37^{\circ} \mathrm{C}$ in a humidified atmosphere of air containing $5 \%$ $\mathrm{CO}_{2}$. All experiments with BALB/c nude mice of 6-7 weeks of age and 20-22 $\mathrm{g}$ of weight were carried out at the Laboratory Animal Center of Tongji University with Institutional Animal Care and Use Committee approval in accordance with institutional guidelines.

Recombinant lentivirus generation and vector construction. The method for creating the recombinant lentivirus package was as previously described (18). An RNAi pLL3.7 (LentiLox 3.7) lentiviral system was used to create lentiviral vectors (Clontech, Beijing, China). For vector pLL3.7-mir145 (pre-miRNA of miR-145 expression element), oligonucleotide pairs for pre-miRNA of miR-145 and linker sequences with $H p a \mathrm{I}$ and XhoI sites were chemically synthesized. The sequences of the oligonucleotides were: top strand, 5'-CGg tta acC ACC TTG TCC TCA CGG TCC AGT TTT CCC AGG AAT CCC TTA GAT GCT AAG ATG GGG ATT CCT GGA AAT ACT GTT CTT GAG GTC ATG GTT ctc gag CG-3'; and bottom strand, 5'-CGe teg agA ACC ATG ACC TCA AGA ACA GTA TTT CCA GGA ATC CCC ATC TTA GCA TCT AAG GGA TTC CTG GGA AAA CTG GAC CGT GAG GAC AAG GTG gtt aac CG-3' (sequences corresponding to miR-145 seed sequences are capitalized and bold, and restriction enzyme sites are lower case and bold) (18). To build the expression plasmid, the pairs of oligos were annealed and inserted into the multiple cloning sites between the HpaI and XhoI sites in the pLL3.7 vector. The negative control plasmid pLL3.7-mir145-Mut was similarly constructed, with the exception that 23 nucleotides in sequences corresponding to
miR-145 were mutated (GTC CAG TTT TCC CAG GAA TCC CT to Gaa Ct Gaa TTa gCA cGA AgC aCT, mutations shown in lower-case). The pLL3.7-mir145 or pLL3.7-mir145-Mut was recombined in the package cell line 293T to create lentiviruses. Recombinant viruses were propagated in $293 \mathrm{~T}$ cells, purified and titered by standard methods, as previously described by our laboratory (21). The corresponding viruses were designated Ldv-mir145 and Ldv-mir145-Mut. Co-transfection of human iPS cells used $4 \times 10^{7}$ PFU/ml Ldv-mir145 or Ldv-mir145-Mut lentivirus according to the manufacturer's instructions. The cells were seeded in a six-well plate in DMEM (Sigma-Aldrich, St. Louis, MO, USA) supplemented with $10 \%$ fetal bovine serum, $100 \mathrm{U} / \mathrm{ml}$ penicillin and $100 \mu \mathrm{g} / \mathrm{ml}$ streptomycin at $37^{\circ} \mathrm{C}$ in a humidified atmosphere of air containing $5 \% \mathrm{CO}_{2}$, until cells were $80 \%$ confluent.

RNA extraction and analysis by quantitative real-time PCR ( $q R T-P C R)$. Total RNA from each cell was isolated using TRIzol reagent (Invitrogen, Carlsbad, CA, USA) according to the manufacturer's instructions. The RNA samples were treated with DNase I (Sigma-Aldrich), quantified and reverse-transcribed into cDNA using the ReverTra Ace- $\alpha$ First Strand cDNA Synthesis kit (Toyobo, Osaka, Japan). qRT-PCR was conducted using a RealPlex4 real-time PCR detection system from Eppendorf Co., Ltd., (Hamburg, Germany), with SYBR Green RealTime PCR Master mix (Toyobo) used as the detection dye. qRT-PCR amplification was performed over 40 cycles with denaturation at $95^{\circ} \mathrm{C}$ for $15 \mathrm{sec}$ and annealing at $58^{\circ} \mathrm{C}$ for $45 \mathrm{sec}$. Target cDNA was quantified using the relative quantification method. A comparative threshold cycle $(\mathrm{Ct})$ was used to determine gene expression relative to a control (calibrator) and steady-state mRNA levels were reported as an $\mathrm{n}$-fold difference relative to the calibrator. For each sample, the marker genes $\mathrm{Ct}$ values were normalized using the formula $\Delta \mathrm{Ct}=\mathrm{Ct}$ _genes-Ct_18S rRNA. To determine relative expression levels, the following formula was used: $\Delta \Delta \mathrm{Ct}=$ $\Delta \mathrm{Ct}$ tall_groups- $\Delta \mathrm{Ct}$ _blankcontrol_group. The values used to plot relative expressions of markers were calculated using the expression $2^{-\Delta \Delta \mathrm{Ct}}$. The mRNA levels were calibrated based on levels of $18 \mathrm{~S}$ rRNA. The cDNA of each gene was amplified using primers as previously described (19).

Methyl-thiazolyl-tetrazolium (MTT) assay for cell proliferation. Each group of JAR and JEG-3 cell lines was seeded at $2 \times 10^{3}$ per well in 96 -well plates and cultured in DMEM supplemented with $10 \% \mathrm{FBS}$ at $37^{\circ} \mathrm{C}$ with $5 \% \mathrm{CO}_{2}$, until cells were $85 \%$ confluent. The MTT (Sigma Chemicals, St. Louis, $\mathrm{MO}$, USA) reagent $(5 \mathrm{mg} / \mathrm{ml})$ was added to the maintenance cell medium at various time points and incubated at $37^{\circ} \mathrm{C}$ for an additional $4 \mathrm{~h}$. The reaction was terminated with $150 \mu \mathrm{l}$ dimethylsulfoxide (DMSO, Sigma Chemicals) per well and the cells were lysed for $15 \mathrm{~min}$, and the plates were agitated every $5 \mathrm{~min}$. Absorbance values were determined using the enzyme linked immunosorbent assay (ELISA) reader (Model 680; Bio-Rad, Hercules, CA, USA) at $490 \mathrm{~nm}$.

Flow cytometric (FCM) analysis of cell cycle by propidium iodide (PI) staining. Each group of JAR and JEG-3 cell lines was seeded at $3 \times 10^{5}$ per well in 6-well plates and cultured until $85 \%$ confluent. Each group of cells was washed with 
PBS three times, then collected by centrifuging (Allegra X-22R; Beckman Coulter, Miami, FL, USA) at 1000 x g for $5 \mathrm{~min}$. The cell pellets were resuspended in $1 \mathrm{ml} \mathrm{PBS}$, fixed in $70 \%$ ice-cold ethanol and kept in a freezer for $>48 \mathrm{~h}$. Prior to FCM analysis, the fixed cells were centrifuged, washed twice with PBS and resuspended in PI staining solution (Sigma Chemicals) containing $50 \mu \mathrm{l} / \mathrm{ml} \mathrm{PI}$ and $250 \mu \mathrm{g} / \mathrm{ml}$ RNase A (Sigma Chemicals). The cell suspension, which was kept in the dark, was incubated for $30 \mathrm{~min}$ at $4^{\circ} \mathrm{C}$ and analyzed by FACS (FCM-500, Beckman Coulter). A total of 20,000 events were acquired for analysis using CellQuest software.

Luciferase report assay. All steps of the luciferase reporter assay were as previously described $(18,22,23)$. NIH-3T3 cells were seeded at $3 \times 10^{4}$ per well in 48 -well plates and co-transfected with $400 \mathrm{ng}$ pLL3.7-mir145, pLL3.7 or pLL3.7-mir145-Mut, 20 ng pGL3cm-Sox2-3UTR-WT or pGL3cm-Sox2-3UTR-Mut, and pRL-TK (Promega, Madison, WI, USA) using Lipofectamine 2000 reagent according to the manufacturer's instructions. Luciferase activity was measured $48 \mathrm{~h}$ after transfection using the dual-luciferase reporter assay system (Promega).

RNA extraction and northern blot analysis. Northern blotting was performed as previously described $(13,24)$. For all groups, $20 \mu \mathrm{g}$ good quality total RNA was analyzed on a $7.5 \mathrm{M}$ urea $12 \%$ PAA denaturing gel and transferred to a Hybond $\mathrm{N}^{+}$nylon membrane (Amersham, Freiburg, Germany). Membranes were crosslinked using UV light for $30 \mathrm{sec}$ at $1,200 \mathrm{~mJ} / \mathrm{cm}^{2}$. Hybridization was performed with the miR-145 antisense starfire probe, 5'-AGG GAT TCC TGG GAA AAC TGG AC-3' (IDT, Coralville, IA, USA), to detect the 22-nt miR-199a fragments according to the manufacturer's instructions. After washing, membranes were exposed for 20-40 h to Kodak XAR-5 films (Sigma-Aldrich). As a positive control, all membranes were hybridized with a human U6 snRNA probe, 5'-GCA GGG GCC ATG CTA ATC TTC TCT GTA TCG-3'. Exposure times for the U6 control probe varied between 15 and $30 \mathrm{~min}$.

Western blot analysis. Total protein extracts from each group of cells were resolved by $12 \%$ SDS-PAGE and transferred on PVDF (Millipore, Billerica, MA, USA) membranes. After blocking, the PVDF membranes were washed 4 times for 15 min with TBST at room temperature and incubated with the primary antibody [rabbit anti-human Sox 2 polyclonal antibody (1:200; Chemicon, Temecula, CA, USA)]. After extensive washing, membranes were incubated with secondary peroxidase-linked goat anti-rabbit IgG (1:1000; Santa Cruz Biotechnology, Inc., Santa Cruz, CA, USA) for $1 \mathrm{~h}$. After washing 4 times for $15 \mathrm{~min}$ with TBST at room temperature, the immunoreactivity was visualized by enhanced chemiluminescence (ECL kit; Pierce Biotechnology, Inc., Rockford, IL, USA) and the membranes were exposed to Kodak XAR-5 films.

Soft agar colony formation assay. The method used was as previously described (25). Soft agar assays were constructed in 6-well plates. The base layer of each well consisted of $2 \mathrm{ml}$ with final concentrations of $1 \mathrm{X}$ medium (DMEM+10\% FBS) and $0.6 \%$ low melting point agarose. The plates were chilled at $4^{\circ} \mathrm{C}$ until solid. Subsequently, a $1.0-\mathrm{ml}$ agar growth layer, consisting of $1 \times 10^{4}$ cells suspended in $1 \mathrm{X}$ media and $0.3 \%$ low melting point agarose, was poured onto the base layer. The plates were again chilled at $4^{\circ} \mathrm{C}$ until the growth layer congealed. Additional $1 \mathrm{X}$ media without agarose $(1.0 \mathrm{ml})$ was added to the top of the growth layer on day 0 and again on day 15 of growth. The cells were allowed to grow at $37^{\circ} \mathrm{C}$ for 1 month and total colonies counted. The assays were repeated a total of 3 times. Results were statistically analyzed by paired t-test using the PRISM Graphpad program (Graphpad Software, La Jolla, CA, USA).

Transwell migration assay. All steps were as previously described (26). The cells $\left(2 \times 10^{5}\right)$ were resuspended in $200 \mu \mathrm{l}$ serum-free medium and seeded on the top chamber of the $6.5 \mathrm{~mm}$ polycarbonate transwell filters $(8.0 \mu \mathrm{m}$ pores; Corning Inc., Corning, NY, USA). The full medium $(600 \mu \mathrm{l})$ containing $10 \%$ FBS was added to the bottom chamber. The cells were allowed to migrate for $24 \mathrm{~h}$ at $37^{\circ} \mathrm{C}$ in a humidified incubator with $5 \% \mathrm{CO}_{2}$. The cells attached to the lower surface of the membrane were fixed in $4 \%$ paraformaldehyde at room temperature for $30 \mathrm{~min}$ and stained with 4,6-diamidino-2-phenylindole (DAPI; C1002; Beyotime Institute of Biotechnology, Jiangsu, China), and the number of cells on the lower surface of the filters was counted under the microscope. A total of 5 fields were counted for each transwell filter.

In vivo xenograft experiments. Logarithmically growing ovarian cancer-initiating cells $\left(\sim 1 \times 10^{5}\right)$ were inoculated into $\mathrm{BALB} / \mathrm{c}$ nude/nude mice. Each experimental group consisted of four mice. After 4 weeks of observation, the mice were sacrificed and tumors were obtained (27). The tumor weight was measured and tumor volume was calculated according to the formula: tumor volume $\left(\mathrm{mm}^{3}\right)=$ length $(\mathrm{mm}) \mathrm{x}$ width $(\mathrm{mm}) \mathrm{x}$ height $(\mathrm{mm})$.

Statistical analysis. Each experiment was performed at least three times and data were expressed as the mean \pm SE. The differences were evaluated using Student's t-tests. $\mathrm{P}<0.05$ was considered to indicate a statistically significant result.

\section{Results}

miR-145 binding with the 3'-UTR sites in Sox2. Using an online research tool, the miRBase Target database (http://www. mirbase.org) $(28,29)$, the precursor miRNA (pre-miRNA) sequences, mature miRNA sequences, chromosomal locations and length of miR-145 and the target gene Sox 2 were analyzed. Seven putative miRNA target sites were identified in the 3'-UTR of Sox2 mRNA, depending on species. This study focused on human miR-145, which targets the human Sox2 3'-UTR, although conservation in this sequence indicates the possibility of binding to varying degrees, across species (Fig. 1). Plasmid DNA encoding each Sox2 mRNA 3'-UTR site [wild-type (wt) Sox2, empty plasmid and mutant Sox2] was co-transfected with the miR-145 expression lentivirus (wt miR-145, empty lentivirus and mutant miR-145 lentivirus) into the mouse embryonic fibroblast cell line NIH-3T3, to examine regulation of Sox 2 gene expression by mature miR-145. The 
A
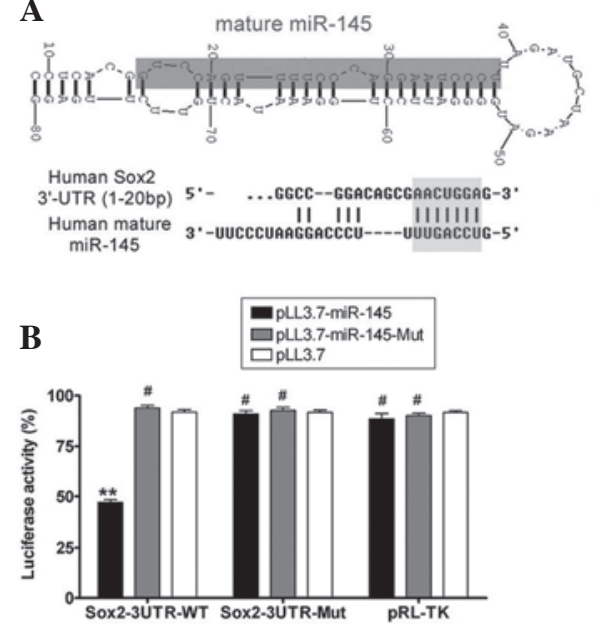

Ca

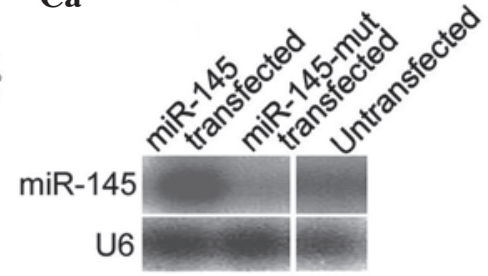

Da

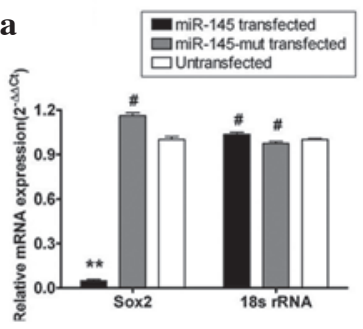

$\mathbf{C b}$

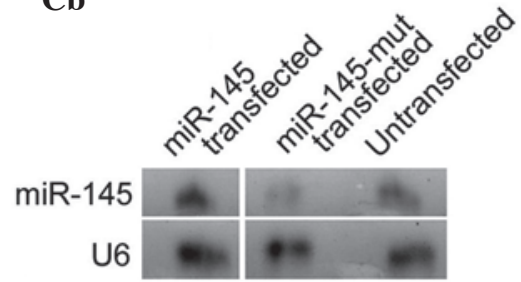

Db

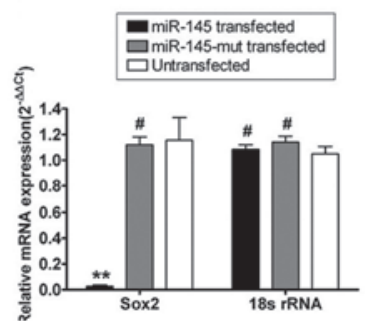

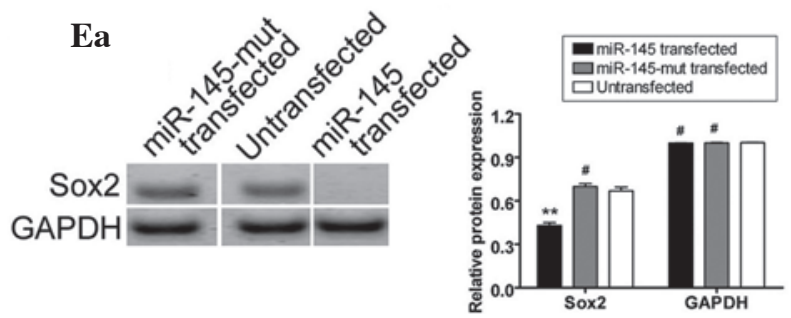

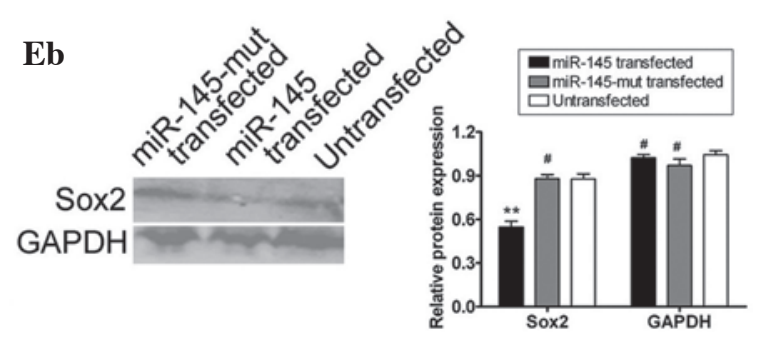

Figure 1. miR-145 and Sox2 expression in different groups. (A) The human Sox2 microRNA (miRNA) 3'-untranslated region (3'-UTR) contains miR-145 binding sites. The mature miR-145 sequences of multiple species were analyzed and contrasted using bioinformatics tools. The typical secondary structure of precursor miRNAs (pre-miRNAs) was compared with miR-145. Pre-miRNA contains stem-loop and hairpin structures and the common binding site is located in an unstable region with a multi-branching loop-like RNA structure. Mature miRNAs are bound in the 3'-UTR of the target gene. Complementarity between miR-145 and the putative human Sox2 3'-UTR site target (1-20 bp downstream) showed that the conserved bases of the putative miR-145 target sequence are present in the human Sox2 3'-UTR. (B) The expression of miR-145 and its interference with the target gene Sox 2 were assessed by luciferase assays. Wild-type (wt) reporter or mutated control luciferase plasmids were transfected into NIH-3T3 cells with miR-145 or mutant miR-145 expression viruses. Luciferase activity within the Sox2 3'-UTR sites was inhibited by miR-145 ( ${ }^{* *} \mathrm{P}<0.01$ vs. pLL3.7; \#P>0.05 vs. pLL3.7; n=3). (C) Northern blot hybridized signals of miR-145 in human choriocarcinoma cells. Northern blot hybridized signals of miR-145 in (Ca) JAR cells and (Cb) JEG-3 cells. Northern blot analysis showed a weaker hybridized signal in mutant miR-145-transfected cells than in wt miR-145-transfected cells. The human U6 probe was used as a loading control. (D) Sox 2 mRNA expression assay in different human choriocarcinoma cells by quantitative real-time PCR (qRT-PCR). qRT-PCR indicated lower expression of Sox 2 mRNA in the (Da) wt miR-145-transfected JAR cells and (Db) wt miR-145-transfected JEG-3 cells than in the corresponding untransfected or mutant miR-145-transfected cells. Relative mRNA expression is shown after normalization to 18S rRNA, which served as an internal control. (E) Western blot showing the expression of Sox 2 in various groups. Western blots showing the expression of Sox 2 in (Ea) JAR cells and (Eb) JEG-3 cells. Sox 2 levels were significantly higher in the untransfected or mutant miR-145-transfected cells than in the miR-145-transfected cells. Data indicate that exogenous miR-145 downregulates Sox 2 expression $\left({ }^{* *} \mathrm{P}<0.01\right.$ vs. untransfected; ${ }^{*} \mathrm{P}>0.05$ vs. untransfected; $\left.\mathrm{n}=3\right)$.

luciferase activity of the Sox2 3'-UTR sites was significantly inhibited by wt miR-145 (Fig. 1), while the luciferase activity of the mutated Sox2 3'-UTR sites was not inhibited, suggesting that Sox2 was targeted by miR-145.

miR-145 specifically influences expression of Sox2 protein in human choriocarcinoma cell lines. Northern blot analysis demonstrated that the hybridized signal of mutant miR-145 in the JAR and JEG-3 choriocarcinoma cell lines was weaker than in cells transfected with wt miR-145. qRT-PCR and western blot analyses were used to determine the effect of exogenous and endogenous miR-145 expression on Sox 2 expression. qRT-PCR analyses revealed decreased Sox 2 mRNA expression in wt miR-145 lentivirus-transfected JAR and JEG-3 cells than in untransfected and mutant miR-145-transfected cells. The relative mRNA expression after normalization to $18 \mathrm{~S}$ ribosomal RNA (rRNA), which served as an internal control, is shown in Fig. 1. Notably, western blotting revealed that Sox 2 levels in untransfected cells (JAR or JEG-3 cell lines) and mutant miR-145 transfected cells (JAR or JEG-3 cell lines) were
$0.667 \pm 0.026$ or $0.876 \pm 0.036$, and $0.669 \pm 0.020$ or $0.879 \pm 0.028$ relative to those of GAPDH, respectively (Fig. 1). These values were significantly higher than those for the wt miR-145 transfected group (JAR: 0.429 \pm 0.019 ; JEG-3: $0.547 \pm 0.040$ relative to GAPDH), which indicated that exogenous miR-145 downregulated Sox 2 expression. Therefore, miR-145 expression may influence endogenous Sox 2 expression.

\section{Proliferation and invasion of human choriocarcinoma cell} lines were inhibited by $\mathrm{miR}$ - 145 . The results of the proliferation assays performed are shown in Fig. 2. Using an MTT assay, the survival rate of wt miR-145 lentivirus-transfected cells was demonstrated to be markedly lower than that of untransfected cells and mutant miR-145-transfected cells at both 3 and 5 days post-transfection. By contrast, no differences in viability were observed in untransfected cells, mutant miR-145-transfected cells and wt miR-145 transfected cells 1 and 2 days posttransfection. The survival rates remained unchanged for untransfected cells and mutant miR-145 transfected cells for the remainder of the time course, which indicated that induced 

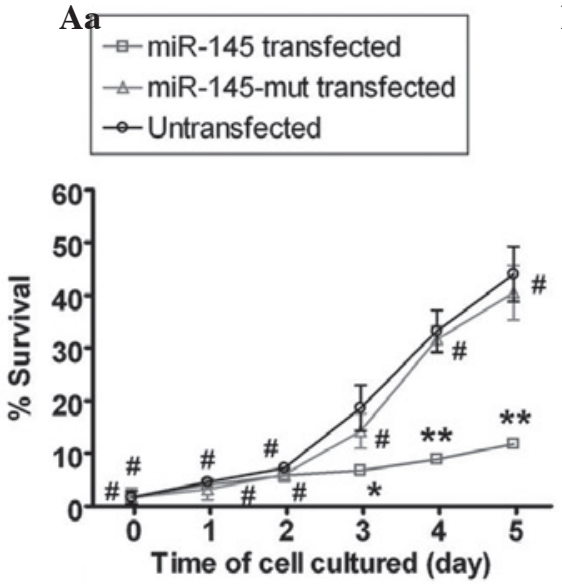

Ab

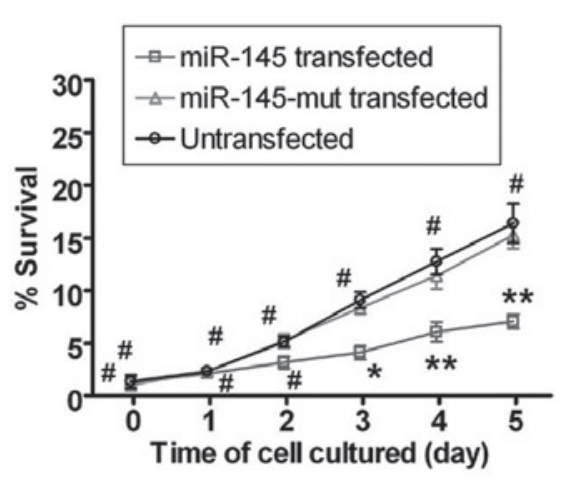

Ba

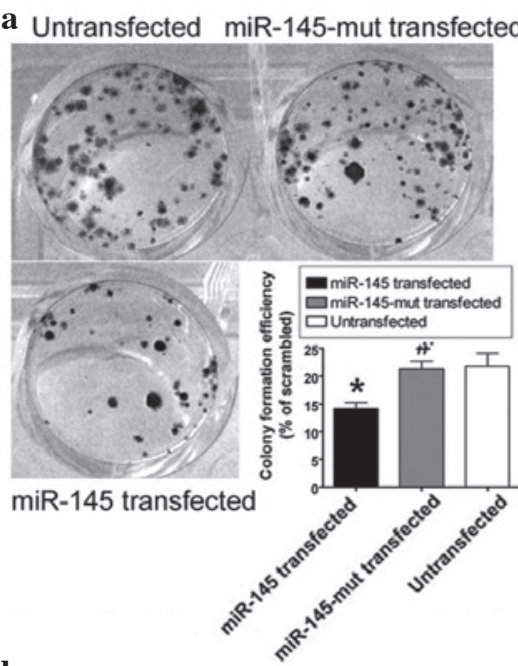

Bb

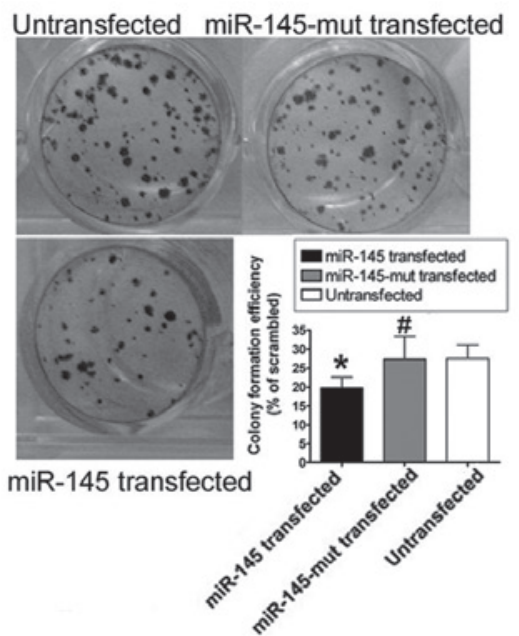

$\mathrm{Ca}$

Untransfected miR-145-mut transfected
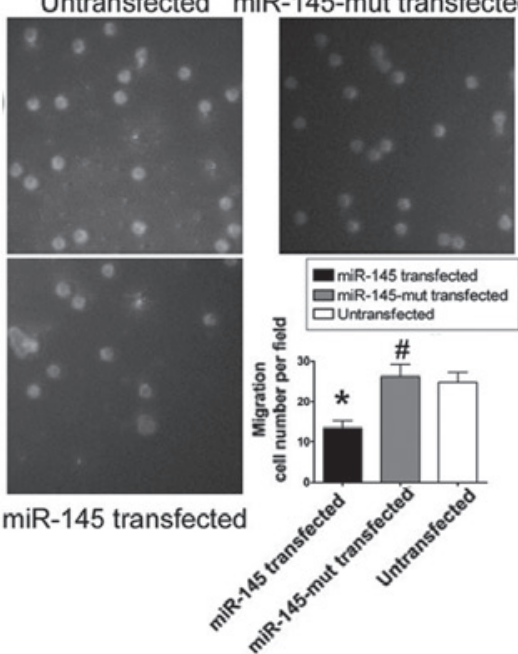

$\mathbf{C b}$

miR-145 transfected

Untransfected miR-145-mut transfected
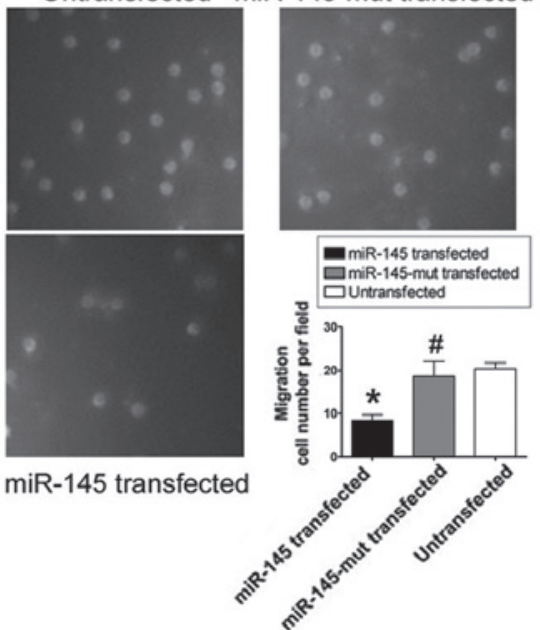

Figure 2. Exogenous miR-145 influences the proliferation and invasion capacity of the human choriocarcinoma cells. (A) Methyl-thiazolyl-tetrazolium (MTT) assays showing the survival rate in various cells. The survival rates of miR-145-transfected (Aa) JAR cells and (Ab) JEG-3 cells were markedly lower than those of the corresponding untransfected and mutant miR-145 transfected cells, at 3 and 5 days post-transfection. ( $\mathrm{P}<0.05$ vs. untransfected cells; ${ }^{* *} \mathrm{P}<0.01$ vs. untransfected cells; ${ }^{~} \mathrm{P}>0.05$ vs. untransfected cells; $\mathrm{n}=3$ ). (B) The results of the transwell migration invasion assay. The numbers of invading cells were significantly lower for miR-145-transfected (Ba) JAR cells and (Bb) JEG-3 cells than for the corresponding untransfected and mutant miR-145-transfected cells. ( $\mathrm{P}<0.05$ vs. untransfected cells; ${ }^{*} \mathrm{P}>0.05$ vs. untransfected cells; $\mathrm{n}=3$ ). (C) The results of soft agar colony formation assay. Soft agar colony formation assays consistently indicated that miR-145-transfected (Ca) JAR cells and (Cb) JEG-3 cells formed substantially fewer colonies than the corresponding control or mutant miR-145-transfected-cells, when plated at low density. (" $\mathrm{P}<0.05$ vs. untransfected cells; ${ }^{\prime \prime} \mathrm{P}>0.05$ vs. untransfected cells; $\mathrm{n}=3$ ). These results suggest that miR-145-mediated repression of Sox 2 expression significantly attenuates the invasion and migration capacity of human choriocarcinoma cells.

exogenous miR-145 expression inhibited the growth of human choriocarcinoma JAR and JEG-3 cell lines in vitro. In addition, migration and invasion ability were shown to be reduced in JAR and JEG-3 cells with stably repressed Sox 2 mediated by miR-145 transfection using transwell migration analysis and soft agar colony formation assays, respectively (Fig. 2). Transwell migration invasion assays showed that the number of invading wt miR-145-transfected JAR cells was significantly lower than the numbers of invading untransfected and mutant miR-145-transfected JAR cells (invading cell numbers: miR-145 transfected group, 13 \pm 2 ; untransfected cells, 24 \pm 2 ; mutant miR-145 transfected cells, 26 \pm 2 ). The results of transwell migration invasion assays in JEG-3 cells were similar to those of JAR cells. These results indicate that the repression of Sox 2 expression by miR-145 significantly attenuates the invasion and migration ability of human choriocarcinoma cells. Soft agar colony formation assays consistently indicated that
miR-145-transfected cells formed substantially fewer colonies compared with controls or mutant miR-145-transfected cells when plated at low density (Fig. 2). In addition, miR-145 transfected, mutant miR-145-transfected and untransfected JAR or JEG-3 cells were stained with PI, and analyzed by flow cytometry to detect changes in cell cycle progression. As shown in Fig. 3, the majority of the wt miR-145-transfected JAR cells were arrested in the $G_{0} / G_{1}$ phase of the cell cycle and the percentage of cells in the $S$ phase were markedly decreased. By contrast, no significant differences were observed in the cell cycle distribution of the mutant miR-145-transfected and untransfected JAR cells. In addition, the majority of wt miR145-transfected JEG-3 cells were arrested in the $G_{0} / G_{1}$ phase of the cell cycle and the percentage of cells in the $G_{2} / M$ phase were markedly decreased. However, no significant differences were observed in the cell cycle distribution of the mutant miR-145-transfected and untransfected cells. The results 
A

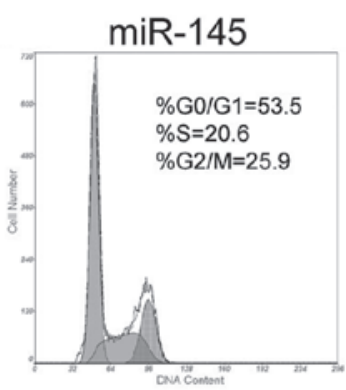

B

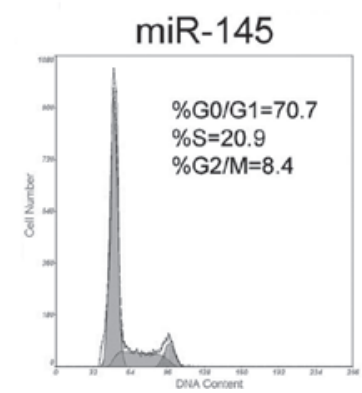

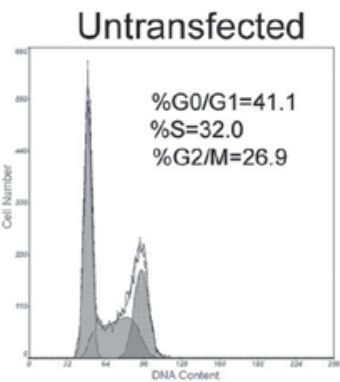
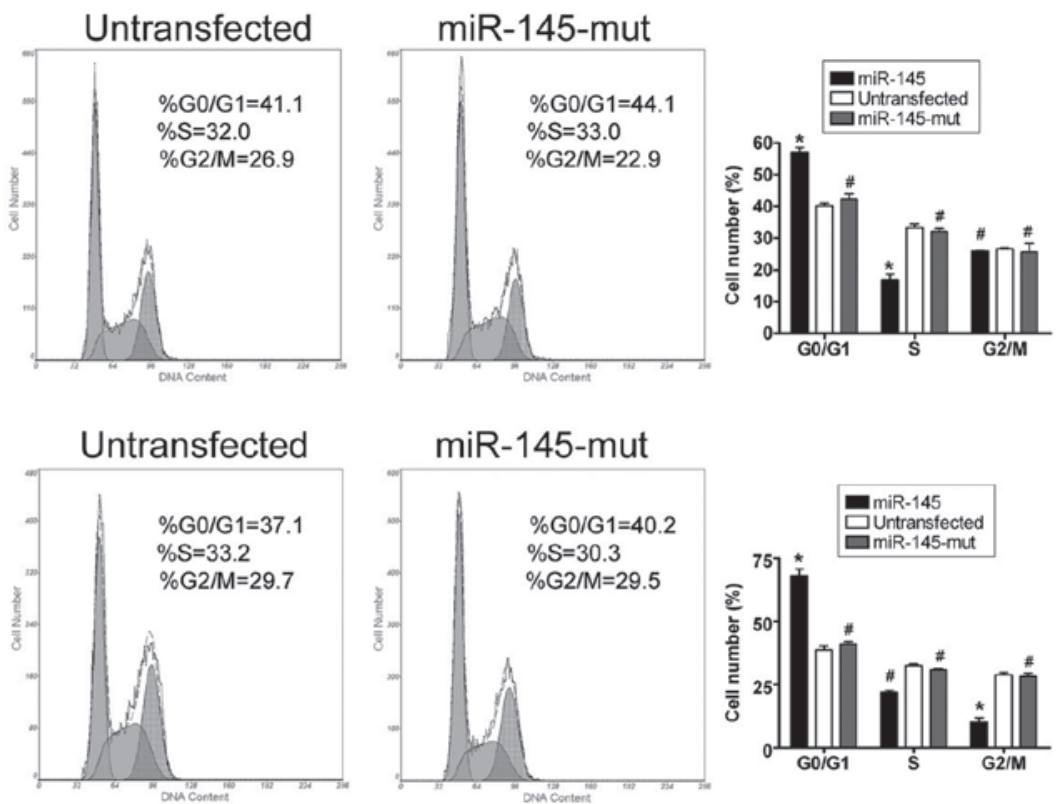

Figure 3. Exogenous miR-145 influences the cell cycle in the human choriocarcinoma cells. (A) Flow cytometric analysis showed that the majority of the miR-145-transfected JAR cells were arrested in the $\mathrm{G}_{0} / \mathrm{G}_{1}$ phase of the cell cycle and the percentage of cells in the $S$ phase was markedly decreased. (B) Flow cytometric analysis showed that the majority of the miR-145-transfected JEG-3 cells were arrested in the $\mathrm{G}_{0} / \mathrm{G}_{1}$ phase of the cell cycle and the percentage of cells in the $\mathrm{G}_{2} / \mathrm{M}$ phases was markedly decreased. ("P< $<0.05$ vs. untransfected cells; \#P>0.05 vs. untransfected cells; $n=3$ ).

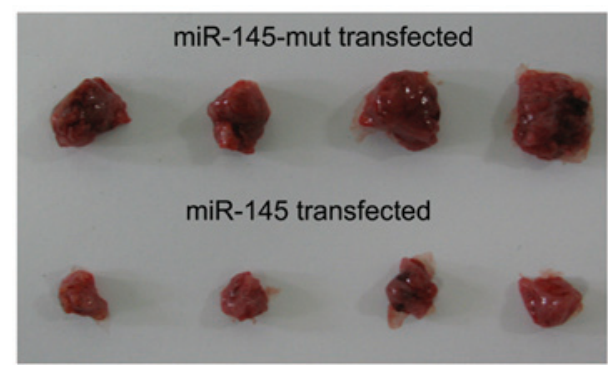

C

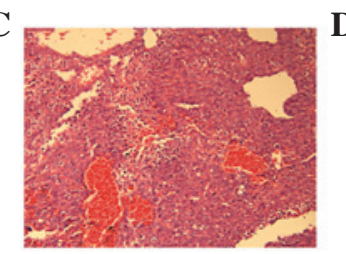

D

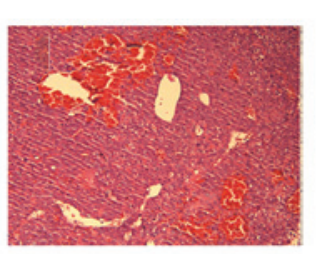

B

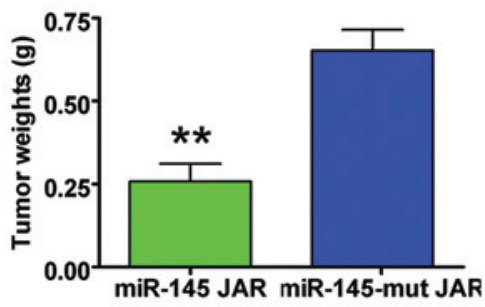

$\mathbf{E}$

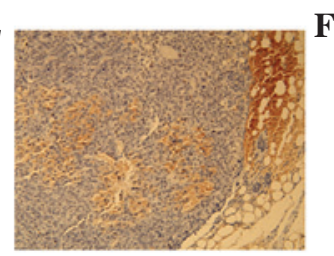

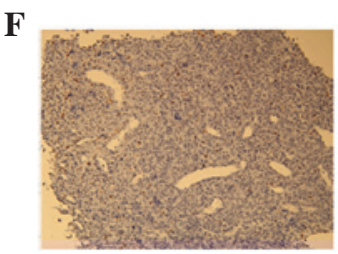

Figure 4. Xenograft experiment results. (A) All mice in the mutant miR-145-transfected group developed tumors $\sim 37$ days after injection, while tumors formed in the miR-145-transfected group grew more slowly. (B) Tumor weights in the miR-145-mut group were heavier than in the miR-145-transfected group, and miR-145 expression was associated with decreased the tumor volume $\left({ }^{* *} \mathrm{P}<0.01\right.$ vs. nontransfected; $\left.\mathrm{n}=4\right)$. Hematoxylin and eosin staining revealed cellular heterogeneity in pathological sections of excised tumor tissue from (C) the mutant miR-145-transfected group and (D) the miR-145-transfected group (original magnification: $\mathrm{x} 200$ ). Immunohistochemistry showing (E) positive or strongly positive Ki-67 staining in tumors formed by mutant miR-145-transfected JAR cells and (F) weakly positive Ki-67 staining in miR-145-transfected cells. Cell proliferation-related protein Ki-67 was a biomarker to evaluate the balance of tumor cell proliferation and programmed cell death (original magnification: $\mathrm{x} 200$ ).

suggested that wt miR-145 expression affected cell cycle regulation in human choriocarcinoma cells in vitro.

Expression of wt miR-145 in JAR cells inhibited subcutaneous tumor growth in nude mice. The effect of miR-145 expression on tumor growth was investigated in vivo by subcutaneous inoculation of the miR-145 lentivirus-transfected JAR cells and mutant miR-145 lentivirus-transfected JAR cells into two groups of nude mice. All the mice in the mutant
miR-145 group developed tumors $\sim 37$ days after injection, whereas tumors were detected in only one in four mice from the miR-145 transfected group at this time. Although both groups developed tumors, the tumors formed by wt miR-145-transfected cells grew more slowly than those in the mutant miR-145-transfected group (Fig. 4). In addition, when the mice were sacrificed 62 days after injection, tumor weights in the mutant miR-145 transfected group were significantly heavier than those in the wt miR-145 lentivirus-transfected 
group. Furthermore, miR-145 expression in the JAR cell line was associated with a significant decrease in tumor volume (Fig. 4). These results suggested that miR-145 expression in the human choriocarcinoma cell line suppressed in vivo tumor growth.

\section{Discussion}

Increasing evidence has shown that miRNA plays an important role in the proliferation and invasion ability of numerous types of cancer cells. However, the miRNAs that regulate human choriocarcinoma cell growth and invasion have not yet been reported. In the present study, it was observed that miR-145 interfered with Sox 2 expression via putative sites located in the 3'-UTR region. Therefore, it was hypothesized that miR-145 suppresses the human choriocarcinoma cell line JAR by downregulation of Sox 2 expression. Putative miRNA target sites in the 3'-UTR of Sox 2 mRNA were used to construct a wt miR-145 expression lentivirus, which was then transfected into the JAR human choriocarcinoma cell line. Luciferase activity assays indicated that the activity of the Sox2 3'-UTR site was significantly inhibited by wt miR-145, while that of the mutated Sox2 3'-UTR site was unchanged, which suggested that miR-145 targeted Sox2. In addition, qRT-PCR and western blot analysis demonstrated that Sox 2 protein expression was reduced in $\mathrm{wt}$ miR-145 lentivirus-transfected JAR cells compared with the levels detected in mutant miR-145 lentivirus-transfected or untransfected cells. FCM analysis revealed that the majority of wt miR-145-transfected cells were arrested in the $G_{0} / G_{1}$ phase of the cell cycle with reduced percentages in the $S$ and $G_{2} / M$ phases, which suggested that miR-145 expression affected the cell cycle regulation of choriocarcinoma cells in vitro. Similarly, exogenous miR-145 expression was shown to inhibit the growth of the JAR cell line in vitro using MTT assays. Soft agar colony formation assay and transwell migration invasion assays showed that the number of invading wt miR-145 transfected cells was significantly lower than the numbers of invading untransfected and mutant miR-145 transfected cells. Finally, xenograft experiments indicated that miR-145 expressed in the JAR cell line also suppressed tumor growth in vivo.

This is in contrast to a previous report which found that the expression of high levels of Sox 2 was associated with malignancy in human lung cancer stem-like cells/cancer-initiating cells (20). In studies of human choriocarcinoma, it was observed that expressed levels of Sox 2 in human gestational trophoblastic neoplasia cells were higher than in the normal trophoblast cells (19). Thus, we considered that there was an association between Sox 2 expression and malignancy in human choriocarcinoma. By contrast, certain studies have also shown that miR-145 may specifically regulate the target gene Sox 2 expression (21). Therefore, we investigated whether the expression of endogenous Sox 2 in human choriocarcinoma was silenced when the miR-145 overexpression might weaken the proliferation and invasion of carcinoma. In the current study, exogenous miR-145 was transfected into the two human choriocarcinoma cell lines, JAR and JEG-3 using a lentiviral system. The effectiveness of miR-145 overexpression not only as an inhibitor of endogenous Sox 2 expression, but also as a suppressor of proliferation and invasion in human choriocarcinoma cell lines, was investigated in several ways, including cell proliferation, invasion and infiltration assays, and tumorigenicity assays in nude mice. The results suggest that not only proliferation, but also invasion and infiltration were reduced following miR-145 overexpression in the human choriocarcinoma cell lines, JAR or JEG-3. In comparison with the wt cell lines, the tumorigenicity in nude mice of JAR cells transfected with exogenous miR-145 was reduced. These results suggest that the proliferation and invasion capacity of human choriocarcinoma cells is associated with Sox 2 expression. It may be speculated that inhibition or loss of miR-145 expression results in excessive Sox 2 expression, and therefore influences tumor growth. Further studies are required to fully elucidate the function of miR-145 in this process. However, it is clear that miR-145 and Sox 2 play potentially important roles in the pathogenesis of human choriocarcinomas.

\section{Acknowledgements}

This study was supported by grants from the Shanghai Committee Medical Science Foundation of China (No.10411967100) to Te Liu and the National Natural Science Foundation of China (31140037) to Zhixue Liu.

\section{References}

1. Bagley RG, Ren Y, Kurtzberg L, et al: Human choriocarcinomas: placental growth factor-dependent preclinical. Int J Oncol 40: 479-486, 2012.

2. Monzo M, Navarro A, Bandres E, et al: Overlapping expression of microRNAs in human embryonic colon and colorectal cancer. Cell Res 18: 823-833, 2008.

3. Kim VN and Nam JW: Genomics of microRNA. Trends Genet 22: 165-173, 2006.

4. Hatfield SD, Shcherbata HR, Fischer KA, Nakahara K, Carthew RW and Ruohola-Baker H: Stem cell division is regulated by the microRNA pathway. Nature 435: 974-978, 2005.

5. Eder M and Scherr M: MicroRNA and lung cancer. N Engl J Med 352: 2446-2448, 2005.

6. Calin GA, Ferracin M, Cimmino A, et al: A MicroRNA signature associated with prognosis and progression in chronic lymphocytic leukemia. N Engl J Med 353: 1793-1801, 2005.

7. Houbaviy HB, Murray MF and Sharp PA: Embryonic stem cell-specific MicroRNAs. Dev Cell 5: 351-358, 2003.

8. Lanza G, Ferracin M, Gafà R, et al: mRNA/microRNA gene expression profile in microsatellite unstable colorectal cancer. Mol Cancer 6: 54, 2007.

9. Chan JA, Krichevsky AM and Kosik KS: MicroRNA-21 is an antiapoptotic factor in human glioblastoma cells. Cancer Res 65: 6029-6033, 2005.

10. Schetter AJ, Leung SY, Sohn JJ, et al: MicroRNA expression profiles associated with prognosis and therapeutic outcome in colon adenocarcinoma. JAMA 299: 425-436, 2008.

11. Bandrés E, Cubedo E, Agirre X, et al: Identification by Real-time PCR of 13 mature microRNAs differentially expressed in colorectal cancer and non-tumoral tissues. Mol Cancer 5: 29, 2006.

12. Volinia S, Calin GA, Liu CG, et al: A microRNA expression signature of human solid tumors defines cancer gene targets. Proc Natl Acad Sci USA 103: 2257-2261, 2006.

13. Zhang L, Liu T, Huang Y and Liu J: microRNA-182 inhibits the proliferation and invasion of human lung adenocarcinoma cells through its effect on human cortical actin-associated protein. Int J Mol Med 28: 381-388, 2011.

14. Chao A, Tsai CL, Wei PC, et al: Decreased expression of microRNA-199b increases protein levels of SET (protein phosphatase $2 \mathrm{~A}$ inhibitor) in human choriocarcinoma. Cancer Lett 291: 99-107, 2010.

15. Tay Y, Zhang J, Thomson AM, Lim B and Rigoutsos I: MicroRNAs to Nanog, Oct 4 and Sox 2 coding regions modulate embryonic stem cell differentiation. Nature 455: 1124-1128, 2008. 
16. Chew JL, Loh YH, Zhang W, et al: Reciprocal transcriptional regulation of Pou5f 1 and Sox 2 via the Oct $4 /$ Sox 2 complex in embryonic stem cells. Mol Cell Biol 25: 6031-6046, 2005.

17. Fong H, Hohenstein KA and Donovan PJ: Regulation of self-renewal and pluripotency by Sox 2 in human embryonic stem cells. Stem Cells 26: 1931-1938, 2008.

18. Xu N, Papagiannakopoulos T, Pan G, Thomson JA and Kosik KS: MicroRNA-145 regulates OCT4, SOX2, and KLF4 and represses pluripotency in human embryonic stem cells. Cell 137: 647-658, 2009.

19. Li AS, Siu MK, Zhang H, et al: Hypermethylation of SOX2 gene in hydatidiform mole and choriocarcinoma. Reprod Sci 15: 735-744, 2008

20. Nakatsugawa M, Takahashi A, Hirohashi Y, et al: SOX2 is overexpressed in stem-like cells of human lung adenocarcinoma and augments the tumorigenicity. Lab Invest 91: 1796-1804, 2011.

21. Liu T, Cheng W, Huang Y, Huang Q, Jiang L and Guo L: Human amniotic epithelial cell feeder layers maintain human iPS cell pluripotency via inhibited endogenous microRNA-145 and increased Sox 2 expression. Exp Cell Res 318: 424-434, 2012

22. Eis PS, Tam W, Sun L, et al: Accumulation of miR-155 and BIC RNA in human B cell lymphomas. Proc Natl Acad Sci USA 102: 3627-3632, 2005.

23. Zhang Y, Chao T, Li R, et al: MicroRNA-128 inhibits glioma cells proliferation by targeting transcription factor E2F3a. J Mol Med (Berl) 87: 43-51, 2009.
24. Cheng W, Liu T, Jiang F, et al: microRNA-155 regulates angiotensin II type 1 receptor expression in umbilical vein endothelial cells from severely pre-eclamptic pregnant women. Int J Mol Med 27: 393-399, 2011.

25. Gupta RA, Shah N, Wang KC, et al: Long non-coding RNA HOTAIR reprograms chromatin state to promote cancer metastasis. Nature 464: 1071-1076, 2010.

26. Jiang F, Liu T, He Y, et al: MiR-125b promotes proliferation and migration of type II endometrial carcinoma cells through targeting TP53INP1 tumor suppressor in vitro and in vivo. BMC Cancer 11: 425, 2011.

27. Carro MS, Lim WK, Alvarez MJ, et al: The transcriptional network for mesenchymal transformation of brain tumours. Nature 463: 318-325, 2010.

28. Kluiver J, Haralambieva E, de Jong D, et al: Lack of BIC and microRNA miR-155 expression in primary cases of Burkitt lymphoma. Genes Chromosomes Cancer 45: 147-153, 2006.

29. Martin MM, Lee EJ, Buckenberger JA, Schmittgen TD and Elton TS: MicroRNA-155 regulates human angiotensin II type 1 receptor expression in fibroblasts. J Biol Chem 281: 18277-18284, 2006. 